\title{
Could Cannabidiol be a treatment for COVID-19-related anxiety disorders?
}

\author{
Saoirse O'Sullivan ${ }^{1}$, Carl Stevenson ${ }^{2}$, and Steven Laviolette ${ }^{3}$ \\ ${ }^{1}$ Artelo Biosciences \\ ${ }^{2}$ University of Nottingham \\ ${ }^{3}$ University of Western Ontario
}

June 22, 2020

\begin{abstract}
COVID-19-related anxiety and post-traumatic stress symptoms (PTSS) or disorder (PTSD) are likely to be a significant longterm issue emerging from the current pandemic. We hypothesise that cannabidiol (CBD), a chemical isolated from Cannabis Sativa with reported anxiolytic properties, could be a therapeutic option for the treatment of COVID-19-related anxiety disorders. In the global over-the-counter CBD market, anxiety, stress, depression and sleep disorders are consistently the top reasons people use CBD. In small randomised, controlled clinical trials, CBD reduces anxiety in healthy volunteers, patients with social anxiety disorder, those at clinical high risk of psychosis, in patients with Parkinson's disease, and in individuals with heroin use disorder. Case reports and series support these findings, extending to patients with anxiety and sleep disorders, Crohn's disease, depression and in PTSD. Preclinical studies reveal the molecular targets of CBD in these indications as the cannabinoid receptors type 1 and 2 (CB1 and CB2) receptors (mainly in fear memory processing), serotonin 5HT1a receptors (mainly in anxiolysis) and peroxisome proliferator-activated receptor gamma (PPAR $\gamma$ ) (mainly in the underpinning anti-inflammatory/anti-oxidant effects). Observational and preclinical data also support CBD's therapeutic value in improving sleep (increased sleep duration/quality and reduction in nightmares) and depression, often comorbid with anxiety. Together these features of CBD to reduce anxiety and depression, and improve sleep disturbances, could be an attractive novel therapeutic option in relieving COVID-related post-traumatic stress symptoms.
\end{abstract}

\section{COVID-related anxiety disorders}

PTSD is a common consequence of major disasters, including epidemics. Epidemiological projections made by the World Health Organisation (WHO) show that post-disaster mental health problems range from mild to very severe distress, affecting between 20 and $50 \%$ of the population. A minority develop new and debilitating mental disorders, and those with pre-existing mental disorders need even more help than before. In the current pandemic, the WHO suggests that levels of loneliness, depression, harmful alcohol and drug use, and self-harm or suicidal behaviour are also expected to rise. Considering the breadth of the COVID-19 pandemic, and the widespread impact of quarantine, social isolation, loss of loved ones (and the inability to grieve socially), loss of income and financial worries, potentially extreme illnesses (which can themselves impact mental health), and frontline working, the current crisis is likely to trigger large numbers of people with long-lasting anxiety and PTSD or post-traumatic stress symptoms (PTSS). In fact, a recent paper suggested 'PTSD as the second tsunami of the SARS-CoV-2 pandemic'.

A systematic review of the evidence on the psychosocial impact of quarantine measures during previous coronavirus outbreaks showed that quarantine measures were consistently associated with negative psychosocial 
outcomes, including depressive symptoms, anxiety, anger, stress, post-traumatic stress, social isolation, loneliness and stigmatisation. For example, the rates of mental health symptoms after the severe acute respiratory syndrome (SARS) outbreak were between 3.7 and $17.3 \%$, and were worst for health care workers. Survey respondents who had been isolated, worked in high-risk workplaces, or had friends or close relatives who contracted SARS were two to three times more likely to develop high levels of PTSS .

In the current COVID-19 outbreak, an early study of residents in Wuhan and surrounding cities were asked about PTSS and sleep qualities. One month after the outbreak (data was collected in Jan 2020), the prevalence of PTSS was about 7\%. A subsequent study (1210 respondents from 194 Chinese cities) found $54 \%$ of respondents rated the psychological impact of the outbreak as moderate or severe; $17 \%$ reported moderate to severe depressive symptoms; $29 \%$ reported moderate to severe anxiety symptoms; and $8 \%$ reported moderate to severe stress levels (Wang et al., 2020). Looking at the mental health effects of COVID-19 in younger people, a cross sectional survey suggests that nearly $15 \%$ of the sample had PTSD symptoms. In patients who contracted COVID-19, the degree of psychological distress may be related to the level of systemic inflammation .

A multinational, multicentre study on the psychological outcomes amongst healthcare workers during COVID-19 surveyed 906 healthcare workers. Using the depression, anxiety and stress scale (DASS-21) scoring system, the authors found anxiety in 16\%, depression in $11 \%$ and stress in $5 \%$ of the study participants. $7 \%$ of the study cohort screened positive for clinical concern of PTSD. Another cross sectional survey of 1257 health care workers treating COVID-19 patients in China found significant reported symptoms of depression (50\%), anxiety (45\%), insomnia (34\%), and distress $(72 \%)$.

These studies highlight the upcoming prevalence of COVID-related anxiety and PTSD, especially amongst healthcare workers and those most directly affected by the pandemic. The pharmacological treatment strategies are likely to involve selective serotonin reuptake inhibitors (SSRIs), selective noradrenaline reuptake inhibitors (SNRIs), serotonin 1A (5HT1A) agonists and benzodiazepines, all of which have side effect profiles that are not always tolerable to patients including drug dependence and withdrawal syndromes. Moreover, these treatments can show limited efficacy in a significant number of patients and their therapeutic effects can have a delayed onset of action (in the case of SSRIs and SNRIs). Psychological treatments (e.g. exposure therapy) are also available but, again, they can have limited or temporary theraputic effects . Thus, novel agents are required to manage these symptoms.

\section{CBD use in anxiety disorders}

Over-the-counter (OTC) CBD products have become very popular in the health and wellness markets globally. One of the features most often proclaimed for CBD is its ability to reduce stress and anxiety. A cross-sectional study of OTC CBD users found that the top three medical conditions for which CBD is used were pain, anxiety and depression. A more recent study found that the top reasons OTC CBD was used were stress relief, relaxation and sleep improvement. A 2020 analysis of social media content revealed that CBD is most discussed as a therapeutic option for anxiety disorders and pain . Supporting the widespread use of OTC CBD for stress and anxiety, there is a growing body of clinical studies demonstrating the anxiolytic effects of CBD across various patient cohorts.

\section{Clinical studies with CBD in anxiety and related disorders}

Several randomised controlled clinical trials and case series report on the ability of CBD to have positive effects on anxiety in healthy volunteers or patients with various diagnoses, and these studies are summarised in Table 2 .

In healthy volunteers in randomised controlled studies, acute doses of 300 or $600 \mathrm{mg}$ given orally reduce the anxiety caused by public speaking, and reduce the blood pressure and cardiovascular response to 
physical and mental stress $(600 \mathrm{mg}$, . Such anxiolytic effects of acute CBD treatment are associated with altered brain activity in corticolimbic regions (e.g. cingulate cortex, amygdala) that mediate cognition and emotional regulation. In healthy volunteers, 7 days of treatment with CBD $(600 \mathrm{mg} /$ day $)$ also reduced the haemodynamic response to physical stress .

In medication-naive patients with generalised social anxiety disorder (SAD), acute oral doses of 300 or $600 \mathrm{mg}$ reduce anxiety caused by public speaking in a randomised, controlled study. Similarly, in teenagers with $\mathrm{SAD}$, a randomised controlled trial showed that 4 weeks CBD (300 mg/day) treatment decreased anxiety measures using the Fear of Negative Evaluation Questionnaire and the Liebowitz Social Anxiety Scale . As with healthy volunteers, the anxiolytic effects of $\mathrm{CBD}$ in anxiety disorders are also linked to alterations in corticolimbic activity . Moreover, in medication-naive participants at clinical high risk of psychosis, CBD (600 mg) partially normalised the alterations in limbic, striatal, and midbrain function in a randomised controlled trial. A follow up study by the same group showed 7 days of treatment with CBD (600 mg/day) in patients at clinical high risk of psychosis partially restored the cortisol response elicited during the Trier Social Stress Test to that observed in the control group . In this study, the experience of anxiety in response to the stress test was greatest in the high-risk patients and lowest in the healthy controls, with those at high risk who were treated with CBD for 7 days exhibiting an intermediate response.

Anxiety is often comorbid with other presentations, and the anxiolytic effects of CBD in other pathologies has also been tested in randomised controlled studies. In patients with Parkinson's disease (PD), a single dose of CBD (300 mg) attenuated the anxiety experimentally induced by a Simulated Public Speaking Test in a randomised, double-blinded, placebo-controlled, crossover trial . In drug-abstinent individuals with heroin use disorder, CBD (either 400 or $800 \mathrm{mg}$ for 3 days) also reduced the anxiety and cortisol responses to the presentation of a drug cue in a double-blind randomized placebo-controlled .

Several case reports also document the anxiolytic effects of CBD. In adults with concerns of anxiety or poor sleep, CBD treatment (25-175 mg per day) improved anxiety and sleep improved for most patients in a recent case series . For a 16-year-old patient with multiple substance use disorder, severe depression, social phobia and narcissistic personality disorder, treatment with CBD (100-600mg/day for 8 weeks) improved depressive and anxiety symptoms. CBD treatment (up to 600mg per day over 19 weeks) in a 14 -year-old patient with Crohn's disease and anxiety disorder (social phobia) reduced the severity of anxiety symptoms . A case report and case series in PTSD patients treated with CBD (2-100 mg/day for 8 weeks) reported improvements in anxiety and sleep quality . A recently published study of an audit of CBD-prescribed patients was completed on 253 patients . Patients with non-cancer pain and mental-health symptoms achieved significant improvements to patient-reported pain, depression and anxiety symptoms.

\section{CBD's mechanism of action in anxiety}

Many preclinical studies have investigated the anxiolytic and mnemonic effects of CBD in animal models of anxiety and learned fear when CBD is administered systemically or directly into the brain, revealing potential mechanisms of action for CBD in these models. Several receptors have been implicated to be molecular sites of action of CBD. The 5HT1A receptor and transient receptor potential vanilloid 1 (TRPV1) channel have been implicated in the anxiolytic effects of CBD . Peroxisome proliferator-activated receptor gamma $(\mathrm{PPAR} \gamma)$ activation is also thought to be involved in the underpinning anti-inflammatory/anti-oxidant effects that help resolve the pathological effects that can lead to anxiety. Activation of the cannabinoid receptor type $1\left(\mathrm{CB}_{1}\right)$ has been implicated in the facilitatory effect of CBD on fear extinction, possibly as an indirect consequence of enhancing endocannabinoids within the brain. Fear extinction is a form of inhibitory learning that suppresses learned fear and forms the theoretical basis for exposure therapy in the treatment of certain anxiety disorders. Interestingly, a study in humans showed that CBD also enhanced fear extinction in healthy volunteers, raising the possibility that CBD might enhance the therapeutic effects of psychological treatments.

In preclinical studies, CBD has also been shown to decrease dopamine (DA) transmission, 5HT transmission 
and to modulate synaptic plasticity in the amygdala and hippocampus . CBD can also block the formation of associative, fear-related memories in specific neural regions, including the nucleus accumbens (NAc) and ventral hippocampus (vHIPP). Thus, reported that direct infusions of CBD into the rodent NAc potently blocked the formation of associative fear memories through a $5 \mathrm{HT}_{1 \mathrm{~A}}$-receptor dependent mechanism. These effects of CBD on fear-memory formation were also dependent on its ability to dampen the activity of DA neurons directly in the ventral tegmental area, an effect that is similarly thought to underlie the putative anti-psychotic properties of CBD . Interestingly, CBD has also been reported to mitigate the anxiogenic effects of delta-9-tetrahydrocannabinol (THC). For example, co-administration of CBD with THC in the rodent vHIPP was shown to block the ability of THC alone to induce potentiation of fear-related memory formation. This effect was dependent on CBD's ability to block hyperstimulation of extracellular-signalrelated kinase 1-2 phosphorylation states (ERK-1-2) induced by THC . Thus, the therapeutic ability of CBD in anxiety and stress management are not reliant on a single molecular target and may involve many biological processes. Accordingly, there is an urgent need to map and validate these preclinical molecular biomarkers associated with CBD's anxiolytic properties onto translational studies in human populations.

\section{CBD and sleep disorders}

As alluded to above, sleep disturbances are often reported in those suffering with anxiety, PTSS and PTSD , and case series suggest sleep disturbances are in improved by CBD in patients with PTSD, particularly in the reduction of nightmares (see Table 3 for a summary of studies that report improvements in sleep with CBD in humans). One placebo-controlled, dose-ranging clinical study found that a dose of $160 \mathrm{mg}$ CBD was effective at improving sleep duration in insomniac volunteers. In preclinical studies, CBD has been shown to block anxiety-related REM sleep alterations . Observational clinical studies investigating the effects of CBD on sleep found CBD (25-300 mg/day) to have positive effects on sleep quality and to decrease sleep disturbances in patients with autism spectrum disorder, Parkinson's, chronic pain and in patients with insomnia .

\section{Current and future approaches for clinical research with CBD in anxiety-related disorders}

Based on this positive background of preclinical data, anecdote and case reports, and small randomised controlled trials, numerous clinical trials are currently registered (see clinicaltrials.gov) assessing the potential of CBD in anxiety-related disorders. There are three phase 2 trials registered assessing the ability of CBD to reduce general anxiety (NCT02548559, NCT04267679, NCT04286594), one phase 3 clinical trial in generalised anxiety disorder (NCT03549819), one phase 2 trial in social anxiety disorder (NCT04086342), three phase 2 trials in PTSD (NCT03518801, NCT03248167, NCT04197102), one phase 3 trial in bipolar depression (NCT03310593), and one phase 1 trial to assess reducing anxiety in Alzheimer's Disease (NCT04075435).

Due to the intrinsic issues hindering the effective use of CBD as an oral drug in the solid-state, such as polymorphism, low solubility and bioavailability, and pharmacokinetic variability, some researchers are looking to improve on the physicochemical characteristics of the CBD molecule. Engineering of the crystalline structure of drug molecules by cocrystalization is a well-developed pharmaceutical strategy of enhanced pharmaceutics . Cocrystals are defined as crystalline materials composed of two or more molecules within the same crystal lattice (FDA Guidance for Industry, 2016). A cocrystal composition of CBD has the potential to overcome numerous physicochemical issues, allowing improved stability and bioavailability. Considerations for selection of the appropriate coformer, to form the CBD cocrystal, for a particular application, such as PTSD, include the properties of inert or pharmacologically active coformers. CBD cocrystal coformers such as tetramethylpyrazine (TMP) that have some evidence of activity in anxiety related disorders or PTSD, may be particularly attractive, and have been patented by Artelo Biosciences for development in this area. 


\section{Summary and conclusion}

Symptoms of anxiety and post-traumatic stress are going to be prevalent as a consequence of the COVID19 pandemic, particularly in those most closely affected by the disease, and in those with existing anxiety conditions. Current anti-anxiety medications possess significant unwanted side-effects, including strong drug dependence and withdrawal syndromes. CBD is already being evaluated as a potential treatment during the active coronavirus disease phase, and we suggest that the preclinical and clinical evidence base supports the hypothesis that CBD could be a novel pharmacological option for treating COVID-related anxiety disorders. CBD could be more preferable compared to some of the medicines currently available with respect to its safety and side effect profile.

\section{Author contribution}

The manuscript was written, reviewed and approved by SOS, CS and SL.

\section{Conflicts of Interest}

SOS is a paid scientific advisor for Artelo Biosciences. CS and SL receive research funding from Artelo Biosciences.

\section{References}

\section{Hosted file}

Table 1.pptx available at https://authorea.com/users/334877/articles/460846-couldcannabidiol-be-a-treatment-for-covid-19-related-anxiety-disorders

Hosted file

Table 2.pptx available at https://authorea.com/users/334877/articles/460846-couldcannabidiol-be-a-treatment-for-covid-19-related-anxiety-disorders

Hosted file

Table 3.pptx available at https://authorea.com/users/334877/articles/460846-couldcannabidiol-be-a-treatment-for-covid-19-related-anxiety-disorders 